\title{
Optimization of weapon-target assignment problem by intuitionistic fuzzy genetic algorithm
}

\author{
Yang Jinshuai a, Li Jin, Wang Yi, Wen Tong, Liu Zhanqiang \\ Air and Missile Defense College, Air Force Engineering University,710051, Xi'an, China
}

\begin{abstract}
Aiming at the shortcoming of precocity and slow convergence in the application of traditional algorithms to solve the Weapon-Target Assignment (WTA) problem, this paper proposed an intuitionistic fuzzy genetic algorithm that combined with simulated annealing Meta-Lamarckian learning strategy and adaptive mutation to improve the efficiency and speed of solving WTA problem. Firstly, it considered the various constraint functions of WTA problem, in which make the threat of remaining targets minimum and the damage from attacks maximum, established the mathematical model. Next, it defined the membership and non-membership functions of object and constraint function, and built the intuitionistic fuzzy WTA model on the basis of the "min-max" operator. Then, this paper designed a strategy of Meta-Lamarckian learning for simulated annealing and adaptive mutation to enhance the capability of local search and the speed of upper convergence for the algorithm. Finally, this method is effective via the simulation and the analysis of comparison with GA, PSO.
\end{abstract}

\section{Introduction}

Weapon target assignment (Weapon-Target Assignment WTA) is a key problem for battlefield command and decision, is a NP complete problem, the size of the solution space with the number of defensive weapons and targets to increase the number of exponential growth. To solve the WTA problem, the heuristic search algorithm ${ }^{[1]}$ such as genetic algorithm (GA), particle swarm optimization (PSO), Yi Qun algorithm (ACO) has a certain effect.

The paper [2] combine the genetic algorithm and ant algorithm to solve the WTA, shorten the reaction time of weapon system, but increase the complexity; the paper [3] proposed a discrete differential evolution algorithm, widely search the solution space to obtain good convergence performance; the paper [4] presented a particle focusing distance adaptive particle swarm algorithm for solving various WTA problem; the paper [5] design a chromosome encoding scheme to meet the constraints, solving the problem into a combinatorial optimization problem without constraints of the form; the paper [6] use genetic algorithm to solve WTA, being fast convergence but can't fundamentally solve the premature convergence of genetic algorithm. Genetic algorithm has the advantages of strong global optimization and fast convergence speed, which can search robustly the WTA problem, but it has a long iterative time and is easy to fall into the local best ${ }^{[7]}$.Aiming at the shortcoming, many scholars embed local search as a kind of learning mechanism into the genetic algorithm to enhance the performance of the algorithm, which depend on the choice of initial point and need to traverse all of the individual, affect learning efficiency and even lead to fall into the local optimum. Therefore, this paper presents an intuitionistic fuzzy genetic algorithm (IFGA) with learning capacity.

\section{Mathematical model of WTA problem}

Assuming $n$ targets, $m$ weapon platforms, the threat coefficient of target $j$ is $V_{j}$, the kill probability of weapon $i$ to kill target $j$ is $p_{i j}(i=1, \mathrm{~K}, n ; j=1, \mathrm{~K}, m)$, and target survival probability is $q_{i j}=1-p_{i j}$, the platform $i$ can launch $W_{i}$ weapons at most, and kill $x_{i j}$ weapons. if $x_{i j}=1$, the target $j$ is assigned to the weapon $i$, else $x_{i j}=0$.The goal of solving WTA problem is to determine the allocation scheme of the weapon platform for the incoming target, which can make the threat of the remaining target minimum and the maximum damage value, so building model:

$$
\begin{gathered}
\min f_{1}(\mathrm{x})=\min \left(\stackrel{a}{j=1}_{j}^{n} V_{j} \mathrm{O}_{i=1}^{m} q_{i j}{ }^{\mathrm{x}_{i j}}\right) \\
\max f_{2}(\mathrm{x})=\max \left(\stackrel{a}{j=1}^{n}\left[1-{ }_{j} \stackrel{\mathrm{O}}{i=1}_{i j} q_{i j}\right]\right)
\end{gathered}
$$

Constraint condition:

1. $\stackrel{a}{j=1}_{j=1}^{n} x_{i j} £ W_{i}:$ the number that weapons platform $i$ 
fire incoming targets $j$ is $W_{i}$ at most;

2. 遝 $x_{i j} £_{i=1}^{m} W_{i}$, the total number of allocated weapons does not exceed the total number of existing weapons;

3. $\stackrel{\mathrm{a}}{j=1}_{n}^{n} V_{j}=1$, for the high threat of target can be assigned many weapons.

Without considering the dynamic factors, in order to meet the three constraints of the number of weapons, the number of enemy targets, the choice of the priority to combat, the objective function is solved, that is to solve the WTA problem.

\section{WTA problem optimization model based on intuitionistic fuzzy sets}

\subsection{Fuzzy WTA problem optimization model}

The WTA problem is a multi-objective and multi constraint problem, show commonly as (2):

$$
\begin{array}{cl}
\min & f_{i}(x), \quad i=1,2, \mathrm{~K} p \\
\text { s.t. } & g_{j}(\mathrm{x}) ? 0, j \quad 1,2, \mathrm{~K}, q
\end{array}
$$

Bellman and Zadeh combine the fuzzy set theory, the formula (2) is transformed into the fuzzy constraint[8], and the mathematical model of the fuzzy optimization problem is established:

$$
\begin{array}{ll}
\text { min } & f_{i}(x), \quad i=1,2, \mathrm{~K} p \\
\text { s.t. } & g_{j}(\mathrm{x}) \% 0, j \quad 1,2, \mathrm{~K}, q
\end{array}
$$

min is shown as fuzzy minimum, $\ell^{\circ}$ is shown as fuzzy less than and equal to. $m_{f_{i}(\mathrm{x})}$ and $m_{h_{i}(\mathrm{x})}$ are defined as fuzzy objective function and constraint function:

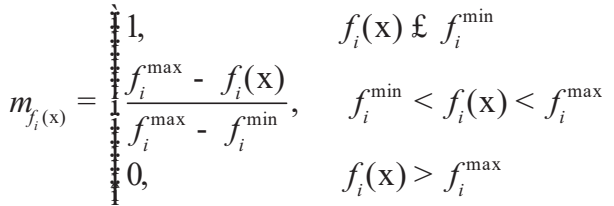

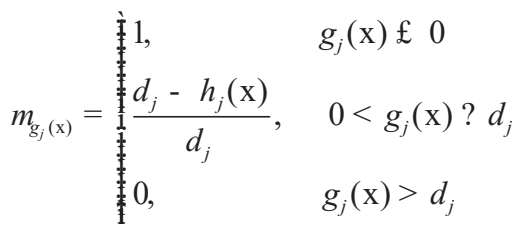

$f_{i}^{\max }$ is maximum value, $f_{i}^{\min }$ is minimum value in the domain $\mathrm{U}, d_{j}$ is maximum deviation of the constraint function. The mathematical model of fuzzy multi-objective optimization is given by the "Min- Max" operator:

$$
\begin{array}{lll}
\max & m \\
\text { s.t. } & m ? m_{f_{i}}(\mathrm{x}), \mathrm{i} & 1,2, \mathrm{~K}, p \\
& m ? m_{g_{j}}(\mathrm{x}), \mathrm{j} & 1,2, \mathrm{~K}, q \\
& 0 \# m_{A}(\mathrm{x}) \quad 1
\end{array}
$$

\subsection{Intuitionistic fuzzy WTA problem optimization model}

$$
f_{i}(\mathrm{x})=\left\{<\mathrm{x}, m_{f_{i}}(\mathrm{x}), g_{f_{i}}(\mathrm{x})>, \mathrm{x} ? U\right\} \text {, which equal to }
$$

the intuitionistic fuzzy objective function, and the non-membership function of the objective function is defined as:

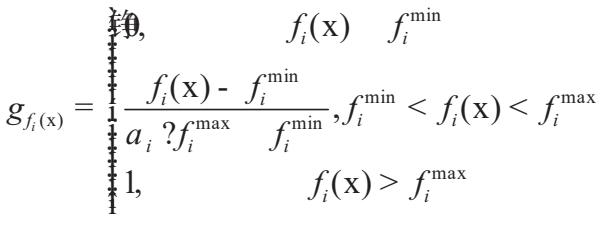

$a_{i}$ could adjust the $p_{f_{i}(x)}$, when $a_{i}=1$, $p_{f_{i}(x)}=0$, and $a_{i}$ 答 $\quad, g_{f_{i}(\mathrm{x})}$ is $0, p_{f_{i}(\mathrm{x})}=1-m_{f_{i}}(\mathrm{x})$. Non-membership dominant optimization at the early stage, to take $a_{i}$ a smaller degree of hesitation, generally $[1,1.3]$

$\mathrm{g}_{j}(\mathrm{x})=\left\{<\mathrm{x}, m_{g_{j}}(\mathrm{x}), g_{g_{j}}(\mathrm{x})>, \mathrm{x} ? U\right\}$, which equal to the intuitionistic fuzzy constraint function, and the non-membership function of the constraint function is defined as:

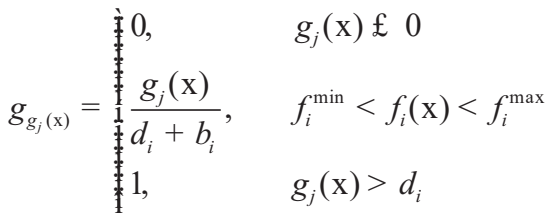

$b_{i}{ }^{3} \quad 0$ could adjust $p_{g_{i}}(\mathrm{x})$, when $b_{i}=0$, $p_{g_{j}}(\mathrm{x})=0 \quad$,and $\quad b_{i}$ 䇣 $\quad, \quad p_{g_{j}}(\mathrm{x}) ? \quad 1 \quad m_{g_{j}}(\mathrm{x})$ Membership dominant optimization at the later stage, to take a smaller degree of hesitation, generally $b_{i}$ take $[0,10]^{[9]}$. A mathematical model of intuitionistic fuzzy multi-objective optimization based on formula (6), (7) and (8):

$$
\begin{array}{llll}
\min & b & \\
\text { s.t. } & b ? & g_{f_{i}}(\mathrm{x}), i & 1,2, \mathrm{~K}, p \\
& b ? & g_{g_{j}}(\mathrm{x}), j & 1,2, \mathrm{~K}, q
\end{array}
$$

Getting"Min"operator from the objective function and the constraint function:

$$
\begin{aligned}
& M=I_{i=1}^{p} f_{i}(\mathrm{x})=\left\{<\mathrm{x}, m_{M_{i}}(\mathrm{x}), g_{M_{i}}(\mathrm{x})>, \mathrm{x} ? U\right\} \\
& S=\mathrm{I}_{j=1}^{q} g_{j}(\mathrm{x})=\left\{<\mathrm{x}, m_{S_{j}}(\mathrm{x}), g_{S_{j}}(\mathrm{x})>, \mathrm{x} ? U\right\}
\end{aligned}
$$

Among, $\quad m_{M}(\mathrm{x})=\mathrm{I}_{i=1}^{p} m_{f_{i}}(\mathrm{x}) \quad, \quad g_{M}(\mathrm{x})={\underset{\mathrm{U}}{i=1}}^{p} g_{f_{i}}(\mathrm{x}) \quad$; $m_{S}(\mathrm{x})=\mathrm{I}_{j=1}^{q} m_{g_{j}}(\mathrm{x}), g_{S}(\mathrm{x})=\stackrel{q}{\mathrm{U}} g_{g_{j}}(\mathrm{x})$.

Getting"Min"operator from the objective function and the constraint function:

$D=M \mathrm{I} S=\left\{\mathrm{x}, m_{D}(\mathrm{x}), g_{D}(\mathrm{x}), \mathrm{x} ? U\right\}$

Among, $m_{D}(\mathrm{x})=m_{M}(\mathrm{x}) \mathrm{I} m_{S}(\mathrm{x}), g_{D}(\mathrm{x})=g_{M}(\mathrm{x}) \cup g_{S}(\mathrm{x})$. Getting "Max" operator in the domain $U$ of intuitionistic fuzzy sets: 


$$
\begin{array}{ll}
\max & m_{D}(\mathrm{x}) \\
\min & g_{D}(\mathrm{x})
\end{array}
$$

Therefore, the form of intuitionistic fuzzy optimization:

$$
\begin{array}{ll}
\max & a-b \\
\text { s.t. } & a £ m_{f}(\mathrm{x}) \\
& a £ m_{g}(\mathrm{x}) \\
& b^{3} g_{f}(\mathrm{x}) \\
& b^{3} g_{g}(\mathrm{x}) \\
& 0 ? a \quad b ? \quad 1
\end{array}
$$

It is clear that $a_{i}=1, b_{i}=0$ in the formula (7), (8), then $a-b=1-2 b$, the intuitionistic fuzzy multi-objective is reduced to the fuzzy multi-objective optimization. Therefore, the mathematical model of WTA and the constraint conditions can be transformed into intuitionistic fuzzy optimization model, which is intuitionistic fuzzy WTA problem, could describe the assignments.

\section{Solving WTA problem based on intuitionistic fuzzy algorithm}

\subsection{Simulated annealing Meta-Lamarck ian learning strategy}

The genetic algorithm iterative evolution mainly through selection, crossover and mutation, only children participate in the competition in every generation, there is no competition between children and parent, leading to lack of excellent individual parent, premature convergence and weak local search ability ${ }^{[10]}$. The simulated annealing algorithm (SA) convergence with probability 1 to the optimal individual ${ }^{[11]}$ in the process of iteration, which can accept the optimal solution, but also can accept inferior solutions in accordance with the Metropolis standards, and with the decrease of temperature, the acceptance probability gradually tends to zero. This is helpful for the algorithm to jump out of local minimum and avoid local optimum. In view of this, this paper adopts simulated annealing Meta-Lamarckian learning strategy ${ }^{[12]}$, the parent of the best individual to join the offspring competition, choose the most suitable for the current search structure for local search from multiple fields in the structure. The steps are as follows:

Step1: uses $n$ different field structures, the best individual in the population local search $n$ (n-1) step based on simulated annealing at the initial temperature;

Step2: calculating value for each field:

$$
\eta_{i}=\left(p f-c f_{i}\right) /(n(n-1))
$$

Among, the $x_{\text {best }}$ before the local search is $p f$, the optimal objective function values obtained by local search of the $\mathrm{i}(\mathrm{i}=1,2, \ldots, \mathrm{n})$ domain structure;

Step3: calculating the probability of choice in various fields according to the reward value $\eta_{i}$ :

$$
p_{u t, i}=\eta_{i} / \sum_{j=1}^{n} \eta_{j}
$$

Step4: Based on the probability of selection, a domain structure is determined by the roulette wheel selection strategy, and then the best individual of the population is searched locally at the current temperature;

Step5: updating bonus value, if the Step4 select the $i$ domain structure, then update the reward value $\eta_{i}=\eta_{i}+\Delta \eta_{i}$, the formula as above is $\Delta \eta_{i}$.

The initial temperature of simulated annealing affects the performance of local search, which uses the function $t_{k+1}=a t_{k}, k=0,1,2, \ldots$ to balance the global and local search speed.

\subsection{Adaptive mutation}

The direction and magnitude of mutation directly affects the efficiency of the algorithm and the convergence speed $^{[15]}$, but the mutation in a GA generally, gene position determined randomly using regular mutation probability, resulting in a large number of similar individuals filled the entire search space at later, reducing the population diversity, slow convergence speed.

This paper adopts adaptive mutation method: to adapt to the variable value is higher than the average fitness of the individual, the mutation probability is low, enabling the individual to protect the next generation; to adapt to the value is lower than the average fitness of individuals, the mutation probability is high, the individual is eliminated. Adaptive mutation probability formula is as follows:

$$
p_{m}=\left\{\begin{array}{cl}
\frac{k_{1}\left(f_{\max }-f\right)}{f_{\text {max }}-f_{\text {avg }}}, & f \geq f_{\text {max }} \\
k_{2}, & f<f_{\max }
\end{array}\right.
$$

$f_{\max }$ is the maximum fitness value of the population, $f_{\text {avg }}$ is the average fitness value of the population, $f$ is the individual fitness value, $k_{1}$ and $k_{2}$ is a constant, and $k_{1}<k_{2}$.

\subsection{IFGA algorithm to solve the WTA problem steps}

The steps that solving the WTA problem by IFGA are as follows:

Step1: initialization. Initialize the population size, the maximum number of iterations to generate the initial population;

Step2: calculating $m_{f_{i}(\mathrm{x})}, m_{h_{j}(\mathrm{x})}, g_{f_{i}(\mathrm{x})}, g_{h_{j}(\mathrm{x})}$ from formula (4), (5), (7), (8)and the fitness value of the individual from formula (14), selecting the best individual;

Step3: calculating learning capacity of the individual and the population, if the individual learning ability is greater than or equal to the average of the population learning ability, then next step, if the average of the 
population, then stop the search;

Step4: using the simulated annealing Meta-Lamarckian learning, selecting and replacing the new generated solution according to the Metropolis standard, and then down the temperature;

Step5: bring the new individual into the iterative evolutionary population, crossover and adaptive mutation;

Step6: end judgment. Whether the algorithm searches for the global optimal value or the maximum number of iterations is to stop, output the optimal allocation scheme, or return to Step2.

\subsection{Time complexity analysis}

The time complexity of the IFGA is analyzed according to the solving steps. The number of chromosomes in the population is $\mathrm{N}, \mathrm{M}$ is the number of iterations, and the iteration time is $\mathrm{T}$. In the standard GA, all the individuals in the population are iterative and the wasted time is $N \cdot M \cdot T$, the time complexity is $O(N \cdot M \cdot T)$. On the basis of the standard GA, the local search strategy and adaptive mutation are added to the IFGA, which increase the wasted time. The probability that the best individual conduct simulated annealing Meta-Lamarckian learning strategy is $P M L_{i}$ (when $P M L_{i}=1$, implementing learning strategies, $P M L_{i}=0$, no learning strategies), the average cost is $T_{P M L}$, then consuming time of IFGA is:

$$
N \cdot M \cdot\left(T+\sum_{i}^{N} P M L_{i} \cdot T_{P M L}\right)
$$

Because the individual of the Meta-Lamarckian learning strategy for simulated annealing is the best individual in the population, the number is small and the time is small, so it is not enough to improve the order of time complexity. Therefore, the complexity of the IFGA is consistent with the standard GA, which does not increase the computational overhead.

\section{Simulation Analysis}

This paper conducted many simulation and calculation by using the Matlab programming language to verify the performance of the algorithm in solving the WTA problem, which achieve a good result. Assuming a total of 10 batches of threat targets, we have 8 different types of weapons platforms can be used to intercept, known to each target threat to us and our success probability of interception of the target, as shown in table 1:

Table 1. Interception probability and threat of targets

\begin{tabular}{ccccccccccc}
\hline \multirow{2}{*}{ Weapon } & \multicolumn{10}{c}{ Target } \\
\cline { 2 - 11 } & 1 & 2 & 3 & 4 & 5 & 6 & 7 & 8 & 9 & 10 \\
\hline 1 & 0.81 & 0.71 & 0.26 & 0.66 & 0.51 & 0.26 & 0.64 & 0.12 & 0.92 & 0.73 \\
2 & 0.73 & 0.14 & 0.83 & 0.55 & 0.12 & 0.82 & 0.57 & 0.59 & 0.71 & 0.38 \\
3 & 0.92 & 0.66 & 0.34 & 0.76 & 0.48 & 0.39 & 0.74 & 0.86 & 0.63 & 0.56 \\
4 & 0.16 & 0.82 & 0.77 & 0.53 & 0.22 & 0.75 & 0.51 & 0.43 & 0.86 & 0.94 \\
5 & 0.51 & 0.49 & 0.85 & 0.90 & 0.35 & 0.76 & 0.84 & 0.59 & 0.61 & 0.46 \\
6 & 0.87 & 0.30 & 0.58 & 0.78 & 0.89 & 0.60 & 0.81 & 0.39 & 0.78 & 0.79 \\
7 & 0.59 & 0.89 & 0.49 & 0.69 & 0.78 & 0.51 & 0.44 & 0.96 & 0.88 & 0.59 \\
8 & 0.84 & 0.69 & 0.78 & 0.92 & 0.54 & 0.81 & 0.59 & 0.67 & 0.32 & 0.66 \\
$V_{j}$ & 0.57 & 0.41 & 0.37 & 0.69 & 0.43 & 0.36 & 0.29 & 0.54 & 0.47 & 0.24 \\
\hline
\end{tabular}

Set the parameters of the IFGA, the number of population is 100 , the maximum number of iterations is $100, k_{1}=0.01, k_{2}=0.02$ which is adaptive mutation probability constant, the initial temperature of 90 , the temperature ratio of $a=0.97$.

The IFGA in the above settings, repeating experiments 20 times, getting the weapon target allocation scheme, as shown in Table 2, the best allocation of benefit value of 3.9365 , takes time $1.068 \mathrm{~s}$.

Table 2. Program of WTA

\begin{tabular}{ccccccccccc}
\hline Target & 1 & 2 & 3 & 4 & 5 & 6 & 7 & 8 & 9 & 10 \\
\hline $\begin{array}{c}\text { Weapo } \\
\mathrm{n}\end{array}$ & 3 & 7 & 5 & 8 & 6 & 2 & 5 & 7 & 1 & 4 \\
\hline \multicolumn{7}{c}{ Comparing }
\end{tabular}

Comparing this paper IFGA in best allocation of benefit value for solving the WTA problem with GA-ACO in [2], DDE-MRR in [3], improved PSO in[4], a GA in [5], the results are shown in figure 1.

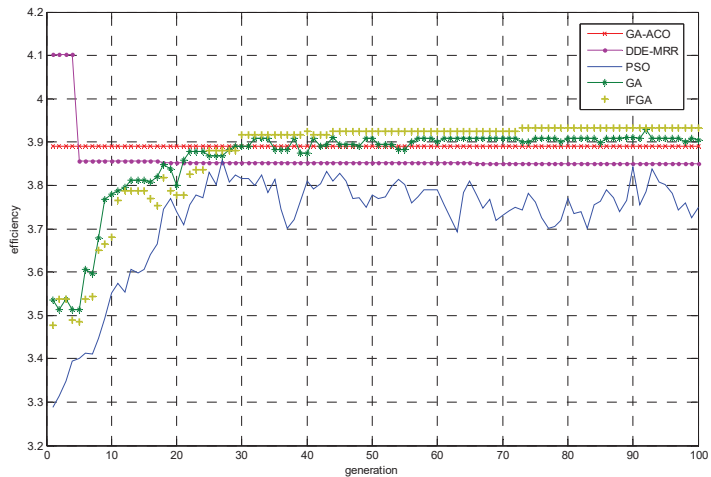

Figure 1. Comparing efficiency that solving WTA

The IFGA achieve the best benefit value of 3.9365 to solve the WTA at 50 iteration; the best benefit GA-ACO achieved with stable value 3.89; the DDE-MMR achieve the best fitness value of 3.86at 60 iteration; the convergence performance of the improved PSO in iterations is poor, it is difficult to get the best benefit value; the best benefit GA to get the value of 3.9365, but 
the convergence effect is poor. The IFGA proposed in this paper is superior to GA-ACO, DDE-MMR and improved PSO. Compared with GA, the IFGA can obtain faster convergence rate and get the best benefit value, thanks to the introduction of non-membership function, clearly describe the solution WTA fitness function; Meta-Lamarckian learning strategy using simulated annealing, local search, jump out of the local minimum, avoid premature; the mutation probability adaptively to balance the global and local search ability to accelerate the convergence speed.
In order to verify the performance of the IFGA in solving WTA problems of large size, simulation of the attacking target and weapon platform respectively $(10,8)$, $(20,16)$ and $(40,20)$, the same intercept probability of success and threat targets, the population size is 100 , the maximum number of iterations is 200 setting, were repeated 20 times, record the maximum and minimum value of the intercept efficiency and average time algorithm is the fastest and the slowest and the average value, and obtain the iterative optimal solution, get the table 3 .

Table 3. Efficiency and time of WTA

\begin{tabular}{ccccccccc}
\hline \multirow{2}{*}{ Target } & \multirow{2}{*}{ Weapon } & \multicolumn{4}{c}{ Intercept Efficiency } & \multicolumn{5}{c}{ Time /s } & \multirow{2}{*}{ Generation } \\
& & Max & Min & Ave & Max & Min & Ave & \\
\hline 10 & 8 & 3.936 & 3.908 & 3.933 & 1.07 & 1.31 & 1.25 & 50 \\
20 & 16 & 7.869 & 7.748 & 7.811 & 1.33 & 1.67 & 1.60 & 80 \\
40 & 20 & 180.05 & 173.42 & 176.97 & 1.582 & 1.883 & 1.785 & 130 \\
\hline
\end{tabular}

It can be found that with the increase of the number of incoming targets and the number of weapons platforms, the interception benefit value will increase, but the change of time is small, only between $2 \sim 3$ s. The IFGA proposed in this paper can effectively solve the problem of large scale WTA, and can meet the requirements of fast and accurate air defense operation.

\section{Conclusions}

It is of great military significance to study the problem of weapon target assignment. In this paper the intuitionistic fuzzy genetic algorithm, by introducing the non-membership function, clearly describes the objectives and requirements of the WTA problem and the constraint conditions, the simulated annealing Meta-Lamarckian learning strategy and adaptive mutation probability, effectively enhance the ability of local search algorithm to speed up the convergence, and to solve the problem of WTA has larger scale good effect.

\section{References}

1. Yu Lianfei, Liu Jin, Zhang Weiming, et al. Review of Weapon-Target Assignment Problem Algorithm [J]. Mathematics in Practice and Theory, 2016, 46(2):26-32.

2. Wu Congmeng, Wang Gongbao. Application of Genetic Ant-Colony algorithm in Target Assignment problem [J]. Ordnance Industry Automation, 2014, 33(4):8-11.

3. Zhang Chunmei, Chen Jie, Xin Bin. A Discrete Differential Evolution Algorithm for the Weapon Target Assignment Problem [J]. Transactions of Beijing Institute of Technology, 2014, 34(3): 289-293.

4. Li Xinran, Fan Yongsheng. An Improved Particle Swarm Algorithm for Weapon Target Assignment Problem Solving $[\mathrm{J}]$. Fire Control \& Command Control, 2014, 39(12):58-61.

5. Wang Wei, Cheng Shuchang, ZHANG Yuzhi. Research on approach for a type of weapon target assignment [J]. Systems Engineering and Electronics, 2008, 30(9):1708-1711.

6. Yang Shanliang, Huang Jian, Liu Yang, et al. Analysis of Weapon Target Assignment Problem in Joint Fire Strike Solving by Genetic Algorithm [J]. Computer Simulation, 2012, 29(3):61-63.

7. Wu Kunhong, Zhan Shixian. Optimization for Target Assignment in Fire Strike Based on Distributed Genetic Simulated Annealing Algorithm [J]. Fire Control \& Command Control, 2016, 41(3):89-92.

8. ANGELOV P P. Optimization in an intuitionistic fuzzy environment [J]. Fuzzy Sets and Systems, 1997, (86): 299-306.

9. Xu Xiaolai, Lei Yingjie, Dai Wenyi. Weighted Intuitionistic Fuzzy Multi-object Programming Based on Improved Particle Swarm Algorithm [J]. Journal of System Simulation, 2009, 21(11):3280-3282.

10. LI Kangping, WANG Pengjun, ZHANG Huihong. The Search of the Best Power Polarity of Ternary FPRM Circuit Based on Simulated annealing Genetic Algorithm[J], Journal of Zhejiang University(Science Edition),2016,43(2):190-194,199.

11. Lin Lingjuan, Liu Xiyu. Rapid partical swarm optimization combined simulated annealing algorithm $[\mathrm{J}]$. Computer Engineering and Applications, 2011, 47(8):27-29.

12. Liu Bo, Wang Ling, Jin Yihui. An effective hybrid PSO-based algorithm for flow shop schedualing with limited buffers [J]. Computers \& Operations Reasearch, 2008, 35(9): 2791-2806.

13. Luan Zhibo, Huang Qitao, Jiang Hongzhou, et al. Mixed application of two learning mechanisms in genetic algorithm [J].Systems Engineering and Electronic, 2009,31(8):1985-1988.

14. Wei Zhen, Wu Lei, Ge Fangzhen, et al. Hybrid PSO Algorithm Based on Memetic Framework [J]. Pattern Recognition and Artificial Intelligence, 2012, 25(2):213-219

15. Zhang Xian, Yaofeng Ren, Wang Runpeng. Research on path planning $[\mathrm{J}]$. Journal of China Ordnance search in continuous time optimal adaptive genetic algorithm based on 2015, 36 (12): 2386-2395. 